\title{
Bromodimethylsulfonium bromide (BDMS) catalyzed synthesis of 2,3-unsaturated- $O$-glycosides via Ferrier rearrangement
}

\author{
Abu T. Khan,* R. Sidick Basha, and Mohan Lal \\ Department of Chemistry, Indian Institute of Technology Guwahati, Guwahati 781 039, India \\ E-mail: atk@iitg.ernet.in
}

\begin{abstract}
This paper is dedicated to my mentor, Prof. Dr. Richard R. Schmidt, on the occasion of his $78^{\text {th }}$ anniversary
\end{abstract}

\begin{abstract}
An expedient synthetic method has been developed for $O$-glycosidation of 3,4,6-tri- $O$-acetyl-Dglucal for the synthesis of 2,3-unsaturated $O$-glycosides using bromodimethylsulfonium bromide (BDMS) as a pre-catalyst through Ferrier rearrangement. Some of the salient features of the present protocol are mild reaction conditions, shorter reaction time, easy work-up procedure, high $\alpha$-selectivity and good yields.
\end{abstract}

Keywords: Ferrier rearrangement, bromodimethylsulfonium bromide (BDMS), 3,4,6-tri- $O$ acetyl-D-glucal, 2,3-unsaturated-O-glycosides

\section{Introduction}

3,4,6-Tri- $O$-acetyl-D-glucal has been found to be useful glycosyl donor for $O$-glycosidic bond formation reaction in carbohydrate chemistry. ${ }^{1}$ In addition, it also serves as a valuable building block for the synthesis of $C$-glycosides ${ }^{2}$ and natural products. ${ }^{3}$ Moreover, the Ferrier rearrangement ${ }^{4}$ has been extensively utilized for the synthesis of 2,3-unsaturated- $O$-glycosides, which are structural subunits of several antibiotics. ${ }^{5}$ They can be further used as glycosyl donors for oligosaccharide synthesis. ${ }^{6}$ Due to their immense potentiality, numerous other synthetic methods have also been developed for the synthesis of 2,3-unsaturated- $O$-glycosides via Ferrier rearrangement using various catalysts such as Lewis acids ${ }^{4}$ or protic acids ${ }^{7}$ or metal salts, ${ }^{8}$ metal triflates $^{9}$ and solid supported heterogeneous catalyst. ${ }^{10}$

Though these methods are quite useful for the synthesis of 2,3-unsaturated- $O$-glycosides in terms of anomeric selectivity, reaction conditions and yield, still there is a further scope to develop a new methodology, which might work even under mild conditions. Some of the drawbacks of the existing methods are: tedious work-up procedure, harsh reaction conditions, 
requirement of expensive catalysts, ${ }^{8,9}$ and involvement of expensive equipment such as microwave. A few years ago, Ye et al. ${ }^{11}$ first demonstrated $O$-glycosidation reaction using a combination of bromodimethylsulfonium bromide and silver triflate. From this successful result, we conceived that bromodimethylsulfonium bromide (BDMS) can be further exploited for $O$ glycosylation reaction since our research group is actively engaged to devise newer synthetic methodologies by employing the same reagent in carbohydrate chemistry. ${ }^{12}$ The unique property and usefulness of bromodimethylsulfonium bromide (BDMS) have been established by our laboratory as well as by others in last few years, which was compiled by us. ${ }^{13}$ Very recently we have shown the same catalyst is also effective for multicomponent reaction for the synthesis of heterocyclic compounds ${ }^{14 \mathrm{a}}$ as well as other organic molecules. ${ }^{14 \mathrm{~b}, \mathrm{c}}$ In this paper, we would like to report the synthesis of 2,3-unsaturated- $O$-glycosides from 3,4,6-tri- $O$-acetyl-D-glucal and various alcohols in presence of BDMS as a pre-catalyst as shown in Scheme 1.

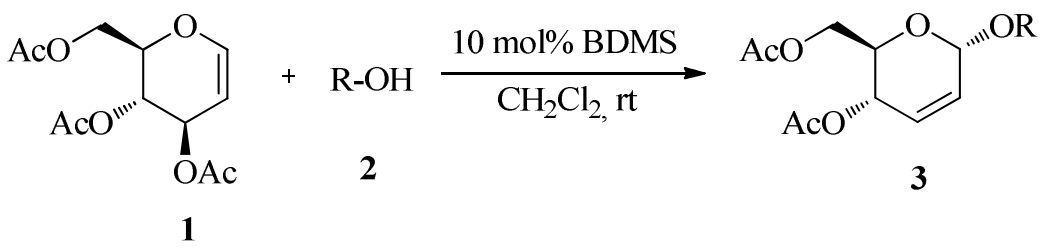

Scheme 1. Synthesis of 2,3-unsaturated-O-glycosides.

\section{Results and Discussion}

For the present study, we have prepared the catalyst BDMS by following the literature procedure. ${ }^{13}$ To find out the optimal reaction conditions, we have chosen as 3,4,6-tri- $O$-acetyl-Dglucal (1) and $p$-bromobenzylalchol (2a) as model substrates. As per our assumption, various reactions were carried out in presence of $5 \mathrm{~mol} \%, 10 \mathrm{~mol} \%$ and $15 \mathrm{~mol} \%$ BDMS in dry DCM, respectively (Table 1, entries 1-3). The desired product 3a was obtained in good yield, which was characterized from ${ }^{1} \mathrm{H}$ NMR, ${ }^{13} \mathrm{C}$ NMR spectra and elemental analysis. To verify the suitability of other solvents, the similar reactions were conducted in $\mathrm{CH}_{3} \mathrm{CN}$, dry ether and mixed solvent system such as DCM/ $\mathrm{CH}_{3} \mathrm{CN}$ (1:1) successively. The successful results are summarized in Table 1 (entries 4-6). To compare the efficacy with other catalysts, similar reactions were performed again with different metal triflates namely $\mathrm{Co}(\mathrm{OTf})_{2}$ and $\mathrm{Mn}(\mathrm{OTf})_{2}$ in acetonitrile (Table 1, entries 7 and 8), which also provided the product 3a in low yield and poor selectivity. The best result was obtained by using $10 \mathrm{~mol} \%$ BDMS in dichloromethane (Table 1, entry 2) at room temperature in terms of reaction time, yield and selectivity. It is noteworthy to mention that on increasing the amount of catalyst, the reaction time reduced, but the yield did not increase significantly. From these results, it is quite clear that BDMS plays a major role in terms of $\alpha$ selectivity and yield. 
Table 1. Optimization of the reaction conditions ${ }^{\text {a }}$

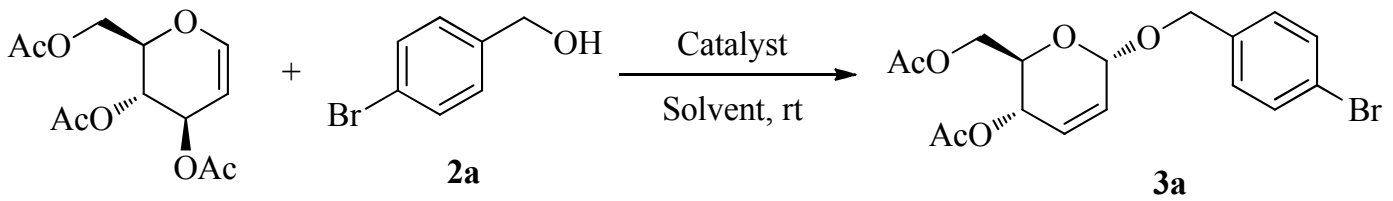

1

\begin{tabular}{ccccc}
\hline Entry & Catalyst & Solvent & Time $(\mathrm{h})$ & $\%$ Yield $^{\mathrm{b}}(\alpha / \beta)^{\mathrm{c}}$ \\
\hline 1 & $5 \mathrm{~mol} \% \mathrm{BDMS}$ & $\mathrm{DCM}$ & 3.5 & $78(5: 1)$ \\
2 & $10 \mathrm{~mol} \% \mathrm{BDMS}$ & $\mathrm{DCM}$ & 2 & $85(7: 1)$ \\
3 & $15 \mathrm{~mol} \% \mathrm{BDMS}$ & $\mathrm{DCM}$ & 1.5 & $83(7: 1)$ \\
4 & $5 \mathrm{~mol} \% \mathrm{BDMS}$ & $\mathrm{CH}_{3} \mathrm{CN}$ & 4 & $72(5: 1)$ \\
5 & $10 \mathrm{~mol} \% \mathrm{BDMS}$ & $\mathrm{Et}_{2} \mathrm{O}$ & 2.5 & $76(6: 1)$ \\
6 & $10 \mathrm{~mol} \% \mathrm{BDMS}$ & $\mathrm{CH}_{3} \mathrm{CN}^{\mathrm{DCCM}}$ & 2.5 & $80(6: 1)$ \\
7 & $5 \mathrm{~mol} \% \mathrm{Co}(\mathrm{OTf})_{2}$ & $\mathrm{CH}_{3} \mathrm{CN}$ & 6 & $56(1: 1)$ \\
8 & $5 \mathrm{~mol} \% \mathrm{Mn}(\mathrm{OTf})_{2}$ & $\mathrm{CH}_{3} \mathrm{CN}$ & 4 & $63(2: 1)$ \\
\hline
\end{tabular}

${ }^{a}$ All the reactions were performed using $1 \mathrm{mmol}$ scale of 3,4,6-tri- $O$-acetyl-D-glucal and 1.1 mmol of $p$-bromobenzyl alcohol at room temperature. ${ }^{\mathrm{b}}$ Isolated yield. ${ }^{\mathrm{c}}$ The ratio $\alpha: \beta$ was determined from the ${ }^{1} \mathrm{H}-\mathrm{NMR}$ spectra.

After optimizing the reaction conditions, a mixture 3,4,6-tri- $O$-acetyl-D-glucal (1) and benzyl alcohol (2b) was treated with $10 \mathrm{~mol} \%$ BDMS under identical reaction conditions and the desired product $\mathbf{3 b}$ was isolated in $92 \%$ yield. By these encouraging results, various reactions were examined with 3,4,6-tri- $O$-acetyl-D-glucal (1) and various alcohols such as 2-phenylethanol (2c), 3-phenylpropanol (2d), 4-phenylbutanol (2e) and 5-phenylpentanol (2f) consecutively in presence of same $10 \mathrm{~mol} \%$ catalyst under similar reaction conditions. The products (3c-f) were obtained from $65 \%$ to $90 \%$ yield, which is mentioned in Table 2 (entries 3-6). Similarly, various alcohols such as primary, secondary, tertiary, allylic and propargylic alcohols were also treated with 3,4,6-tri- $O$-acetyl-D-glucal (1) consecutively in presence of $10 \mathrm{~mol} \%$ BDMS under identical reaction conditions (Table 2, entries 7-14). The yield, reaction time and ratio of the products are depicted in Table 2. All the products were characterized by measuring specific rotation, ${ }^{1} \mathrm{H}$ $\mathrm{NMR},{ }^{13} \mathrm{C}$ NMR and elemental analysis. In addition, the structure of product $3 \mathbf{n}$ was further confirmed by single-crystal X-ray data, which is shown in Figure 1. It is important to mention 
that the alcohols namely cetyl alcohol (2i), tert-butyl alcohol (2l), propargyl alcohol (2n) provide exclusively the $\alpha$ anomer of the 2,3-unsaturated- $O$-glycosides under the experimental conditions.

Table 2. BDMS catalyzed Ferrier rearrangement of 3,4,6-tri- $O$-acetyl-D-glucal with alcohols

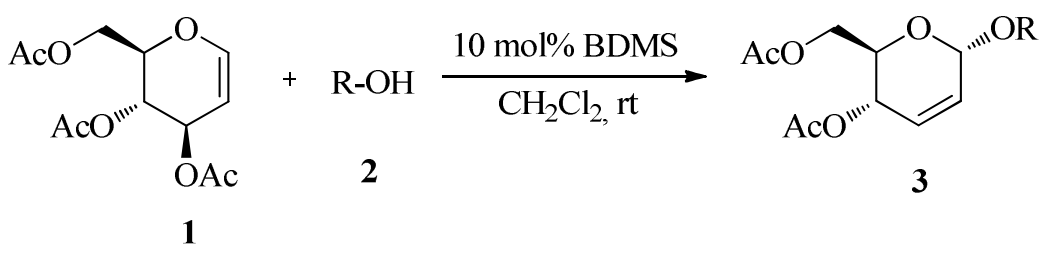

(2)


Table 2 (continued)

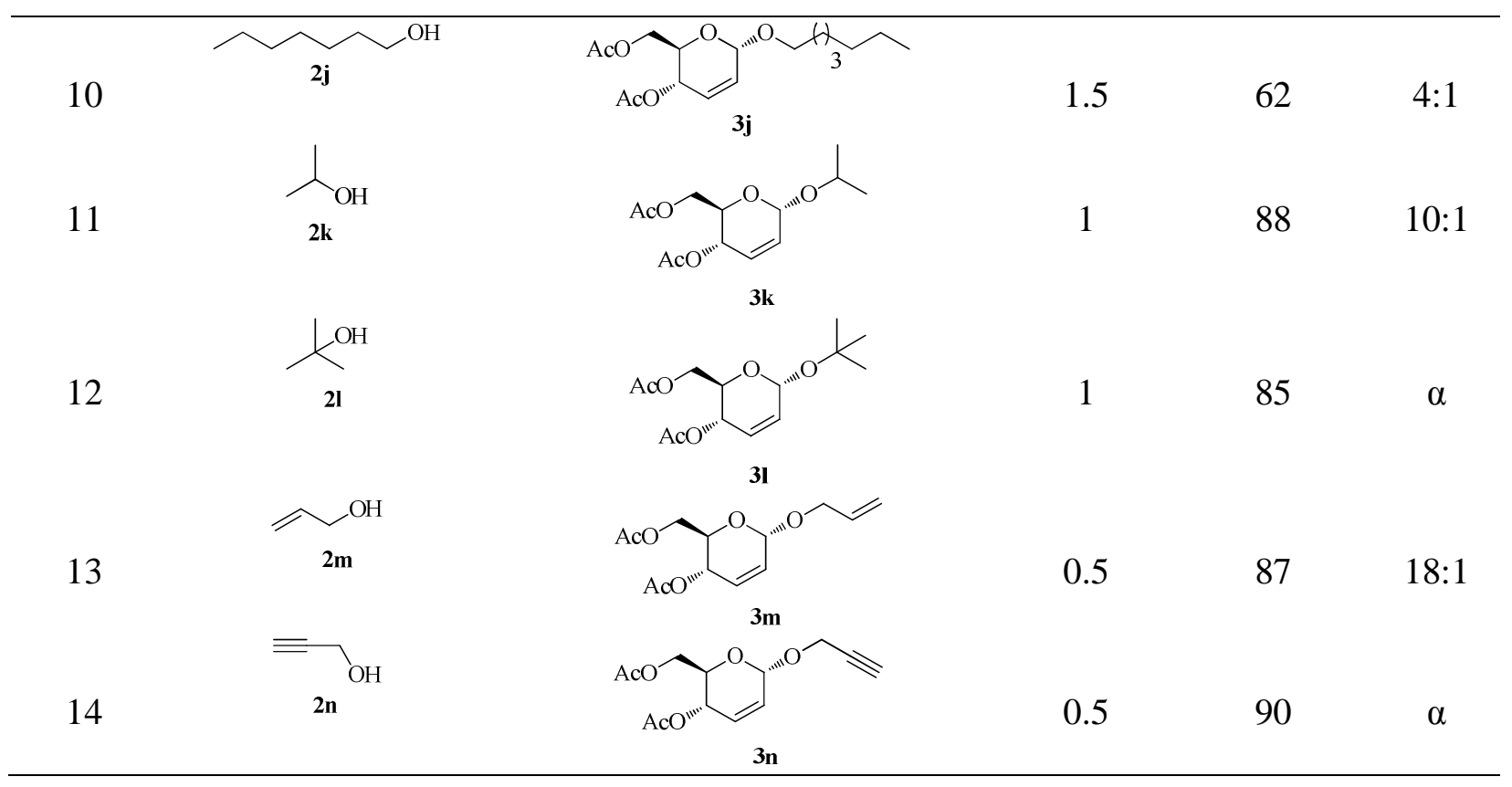

${ }^{a}$ All the reactions were carried out with $1 \mathrm{mmol}$ 3,4,6-tri- $O$-acetyl-D-glucal, $1.1 \mathrm{mmol}$ alcohol using 10 mol\% BDMS as a catalyst. ${ }^{b}$ Isolated yield. ${ }^{c}(\alpha / \beta)$ ratio was determined by anomeric proton integration from ${ }^{1} \mathrm{H}-\mathrm{NMR}$ spectra.

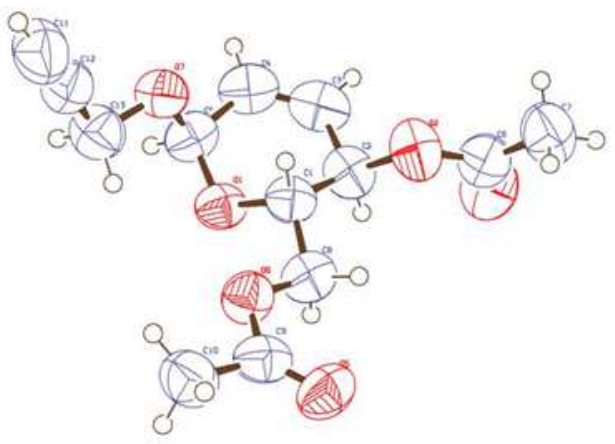

Figure 1. ORTEP diagram of 3n (CCDC no: 875412).

The formation of products can be explained as follows: Initially BDMS reacts with alcohol to form dry $\mathrm{HBr}$ and the intermediate $\mathbf{A},\left[\mathrm{Me}_{2} \mathrm{~S}^{+} \mathrm{OR}\right] \mathrm{Br}^{-}$. The in situ generated $\mathrm{HBr}$ assists the formation of the intermediate cyclic allylic oxocarbenium ion (B) from 3,4,6-tri- $O$-acetyl-Dglucal, which was originally proposed by Ferrier. ${ }^{4 \mathrm{a}}$ Then, alcohol adds with the intermediate $\mathbf{B}$ in a quasi-axial fashion preferentially to provide the product 2,3-unsaturated- $O$-glycosides $\mathbf{3}$ having major $\alpha$-selectivity as shown in Scheme 2. To prove the efficacy of BDMS as compared to 
aqueous $\mathrm{HBr}$, a similar reaction was performed with a mixture of 3,4,6-tri- $O$-acetyl-D-glucal (1) and benzyl alcohol (2b) in presence of $48 \%$ aqueous $\mathrm{HBr}(0.02 \mathrm{~mL})$ at room temperature. Very poor conversion was noted and $30 \%$ yield of $\mathbf{3 b}$ (based on starting material recovery) was obtained. We did not observe formation of any hydrated product while reaction is carried out with $48 \%$ aqueous $\mathrm{HBr}$. In addition, we have also noted that a large scale reaction e.g. $10 \mathrm{mmol}$ scale can be done in presence of $5 \mathrm{~mol} \%$ catalyst. The efficiency and generality of the present protocol can be visualized at a glance by comparing our results with some of the other recently reported procedures (as shown in Table 3). The results have been compared with respect to reaction time, catalyst used and yields. From the comparative results, we may conclude that our protocol is equally efficient in comparison to the earlier reported methods.

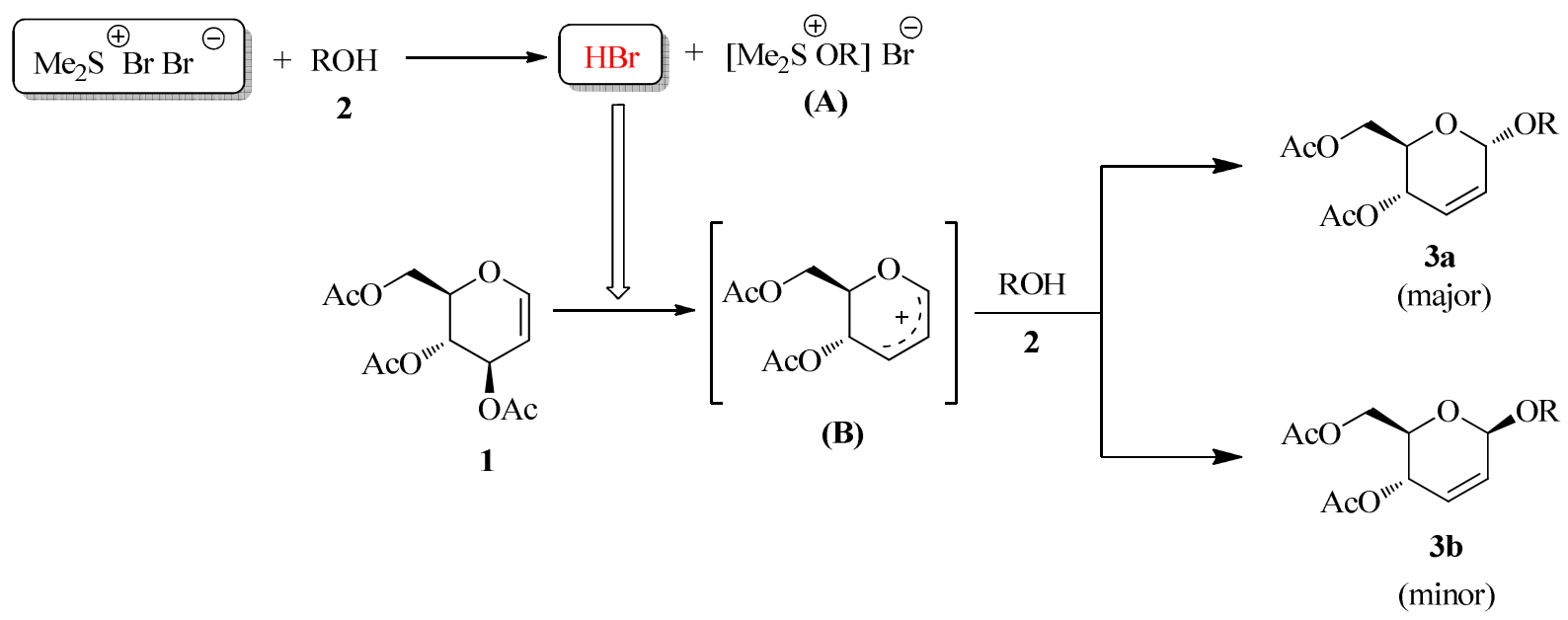

Scheme 2. Plausible mechanism for the formation of 2,3-unsaturated- $O$-glycosides.

Table 3. Comparison of BDMS catalyzed Ferrier rearrangement with the existing reported method of 3,4,6-tri- $O$-acetyl-D-glucal with different alcohols

\begin{tabular}{|c|c|c|c|c|c|c|}
\hline S.No & Product obtained & Catalyst used & Time (h) & Yield $(\%)^{\mathrm{b}}$ & $(\alpha / \beta)^{\mathrm{c}}$ & Ref \\
\hline 1 & $3 \mathbf{b}\left(\mathrm{R} . \mathrm{CH}_{2} \mathrm{Ph}\right)$ & $\begin{array}{c}\mathrm{BDMS} \\
\mathrm{NaHSO}_{4} \\
\mathrm{Fe}_{2}\left(\mathrm{SO}_{4}\right)_{3} \cdot \mathrm{XH}_{2} \\
\mathrm{O}\end{array}$ & $\begin{array}{c}0.5 \\
5 \mathrm{~min} \\
1.5\end{array}$ & $\begin{array}{l}92 \\
91 \\
85\end{array}$ & $\begin{array}{c}8: 1 \\
4: 1 \\
-\end{array}$ & $\begin{array}{c}- \\
8 b \\
7 e\end{array}$ \\
\hline 2 & 3c $\left(\mathrm{R}^{\left.-\mathrm{CH}_{2} \mathrm{CH}_{2} \mathrm{Ph}\right)}\right.$ & $\begin{array}{l}\text { BDMS } \\
\mathrm{H}_{3} \mathrm{PO}_{4}\end{array}$ & $\begin{array}{c}0.5 \\
15 \mathrm{~min}\end{array}$ & $\begin{array}{l}90 \\
92\end{array}$ & $\begin{array}{l}9: 1 \\
8: 1\end{array}$ & $\begin{array}{l}- \\
7\end{array}$ \\
\hline
\end{tabular}


Table 3 (continued)

\begin{tabular}{|c|c|c|c|c|c|c|}
\hline S.No & Product obtained & Catalyst used & Time (h) & Yield $(\%)^{\mathrm{b}}$ & $(\alpha / \beta)^{\mathrm{c}}$ & Ref \\
\hline \multirow{3}{*}{3} & \multirow{3}{*}{$3 \mathbf{g}\left(\mathrm{R} . n-\mathrm{C}_{4} \mathrm{H}_{9}\right)$} & BDMS & 0.5 & 86 & $6: 1$ & - \\
\hline & & $\mathrm{NaHSO}_{4}$ & 1 & 80 & $7: 1$ & $10 \mathrm{~b}$ \\
\hline & & $\mathrm{H}_{3} \mathrm{PO}_{4}$ & $10 \mathrm{~min}$ & 93 & $8: 1$ & 7 \\
\hline \multirow{2}{*}{4} & \multirow{2}{*}{ 3k $\left(\mathrm{R} \mathrm{CHMe} \mathrm{HH}_{2}\right)$} & BDMS & 1 & 88 & $10: 1$ & - \\
\hline & & $\mathrm{NbCl}_{5}$ & $1.5 \mathrm{~min}$ & 85 & $4: 1$ & $8 \mathrm{f}$ \\
\hline \multirow{4}{*}{5} & \multirow{4}{*}{$\mathbf{3 m}\left(\mathrm{R} \cdot \mathrm{CH}_{2} \mathrm{CH}=\mathrm{CH}_{2}\right)$} & BDMS & 0.5 & 87 & $18: 1$ & - \\
\hline & & $\mathrm{NaHSO}_{4}$ & $5 \min$ & 72 & $9: 1$ & $10 \mathrm{~b}$ \\
\hline & & $\mathrm{ZnCl}_{2} / \mathrm{Al}_{2} \mathrm{O}_{3}$ & $20 \mathrm{~min}$ & 83 & $\alpha$ & $10 \mathrm{~d}$ \\
\hline & & $\mathrm{NbCl}_{5}$ & $2.0 \mathrm{~min}$ & 95 & $4: 1$ & $8 f$ \\
\hline \multirow{4}{*}{6} & \multirow{4}{*}{ 3n $\left(\mathrm{R} . \mathrm{CH}_{2} \mathrm{C} \equiv \mathrm{CH}\right)$} & BDMS & 0.5 & 90 & $\alpha$ & - \\
\hline & & $\mathrm{Bi}(\mathrm{OTf})_{3}$ & $5 \mathrm{~min}$ & 73 & $\alpha$ & $9 \mathrm{~d}$ \\
\hline & & $\mathrm{ZnCl}_{2} / \mathrm{Al}_{2} \mathrm{O}_{3}$ & $10 \mathrm{~min}$ & 88 & $\alpha$ & $10 \mathrm{~d}$ \\
\hline & & $\mathrm{NbCl}_{5}$ & 2.5 & 92 & $4: 1$ & $8 \mathrm{f}$ \\
\hline
\end{tabular}

\section{Conclusions}

In conclusion, we have devised a simpler and an much efficient synthetic protocol for the synthesis of 2,3-unsaturated- $O$-glycosides from 3,4,6-tri- $O$-acetyl-D-glucal and alcohols using BDMS as a catalyst via Ferrier rearrangement. The significant features of the present protocol are: high $\alpha$-selectivity, simplicity, inexpensive catalyst, non-aqueous work-up, compatibility with wide range of substrates and good yields. Further study on 2,3-unsaturated- $O$-glycosides for the synthesis of oligosaccharide with biological relevance is undergoing in the laboratory, which will be reported in due time.

\section{Experimental Section}

General. Analytical thin-layer chromatography was performed on precoated TLC plates (0.2 mm layer thickness of silica gel 60 F-254). Spot were visualized by spraying MOSTAIN solution 
(by dissolving $20 \mathrm{~g}$ ammonium heptamolybdate and $0.4 \mathrm{~g}$ cerium(IV) sulfate in $400 \mathrm{~mL}$ of $10 \%$ $\mathrm{H}_{2} \mathrm{SO}_{4}$ solution). Column chromatography was carried out using silica gel (60-120 mesh, Merck or Qualigen). Solvent was removed using a rotary evaporator. Optical rotations were measured with a Perkin-Elmer 243 polarimeter at $25{ }^{\circ} \mathrm{C}$ temperature. Melting points were determined on a Büchi melting point apparatus and are uncorrected. IR spectra were recorded on a Perkin-Elmer Spectrum One FT-IR spectrometer. ${ }^{1} \mathrm{H}$ and ${ }^{13} \mathrm{C}$ NMR spectra were recorded on a Varian (400 $\mathrm{MHz}$ ) spectrometer using TMS as internal standard. Chemical shifts reported in parts per million (ppm). ${ }^{1} \mathrm{H}$ NMR data are reported in the order of chemical shift, multiplicity (s.singlet, d.doublet, t.triplet, dd.doublet of doublet, m.multiplet), coupling constant in hertz (Hz) and number of protons. Elemental analysis was performed using a Perkin-Elmer CHNS/O-2400 Series II elemental analyzer.

General experimental procedure. To a stirred mixture of 3,4,6-tri- $O$-acetyl-D-glucal (1 equiv.) and alcohol (1.1equiv.) in dry dichloromethane $(2 \mathrm{~mL})$ was added $10 \mathrm{~mol} \%$ of BDMS $(0.023 \mathrm{~g})$ at room temperature. The reaction mixtures were stirred for the required time (Table 2) and the reactions were monitored by TLC till the completion of reaction. The solvent was evaporated in a rotary evaporator and the crude residues were passed through a silica gel column to get the desired pure products. The products were eluted with ethyl acetate: hexane mixture (1:9).

4-Bromobenzyl 4,6-di- $\boldsymbol{O}$-acetyl-2,3-dideoxy- $\alpha$-D-erythro-hex-2-enopyranoside $(3 \mathrm{3a}) \cdot[\alpha]_{\mathrm{D}}{ }^{25}$ $+47^{\circ}$ (c 0.20, $\mathrm{CH}_{2} \mathrm{Cl}_{2}$ ); IR (KBr): 3456, 2924, 1742, 1488, 1369, 1231, 1069, 1038, 1010, 968, $803 \mathrm{~cm}^{-1} ;{ }^{1} \mathrm{H}$ NMR $\left(400 \mathrm{MHz}, \mathrm{CDCl}_{3}\right): \delta 7.48(\mathrm{~d}, J .8 .4 \mathrm{~Hz}, 2 \mathrm{H}), 7.23(\mathrm{~d}, J .8 .4 \mathrm{~Hz}, 2 \mathrm{H}), 5.91(\mathrm{~d}$, J.10.4 Hz, 1H), 5.86-5.82 (m, 1H), 5.33 (dd, J.10 Hz, J.1.2 Hz, 1H), 5.11 (s, 1H), 4.75 (d, J.12.4 $\mathrm{Hz}, 1 \mathrm{H}), 4.56-4.53(\mathrm{~m}, 1 \mathrm{H}), 4.24$ (dd, J.12 Hz, J.5.6 Hz, 1H), 4.16-4.10 (m, 2H), 2.09 (s, 3H), $2.08(\mathrm{~s}, 3 \mathrm{H}) ;{ }^{13} \mathrm{C} \mathrm{NMR}\left(100 \mathrm{~Hz}, \mathrm{CDCl}_{3}\right): \delta 170.9,170.5,136.8,131.8,129.8,129.6,127.7$, 121.9, 93.8, 69.6, 67.3, 65.4, 63.0, 21.1, 20.9. Anal. Calcd. for $\mathrm{C}_{17} \mathrm{H}_{19} \mathrm{BrO}_{6}(399.23): \mathrm{C}, 51.14 ; \mathrm{H}$, 4.80. Found: $\mathrm{C}, 50.98 ; \mathrm{H}, 4.70 \%$.

Benzyl 4,6-di- $O$-acetyl-2,3-dideoxy- $\alpha$-D-erythro-hex-2-enopyranoside $(3 \mathrm{~b}) \cdot[\alpha]_{\mathrm{D}}{ }^{25}+66^{\circ}(c$ 0.13, $\mathrm{CH}_{2} \mathrm{Cl}_{2}$ ); IR (KBr): 3031, 2903, 1746, 1497, 1454, 1405, 1371, 1236, 1186, 1102, 1041, 969, 909, 734, 699, $605 \mathrm{~cm}^{-1}$; ${ }^{1} \mathrm{H}$ NMR (400 MHz, $\left.\mathrm{CDCl}_{3}\right): \delta$ 7.35-7.27 (m, 5H), 5.90-5.82 (m, 2H), 5.33 (dd, J.1.6 Hz, J.2.8 Hz, 1H), $5.13(\mathrm{~s}, 1 \mathrm{H}), 4.80$ (d, J.11.6 Hz, 1H), 4.62-4.57 (m, 1H), 4.30-4.22 (m, 1H), 4.17-4.08 (m, 2H), $2.08(\mathrm{~s}, 3 \mathrm{H}), 2.06(\mathrm{~s}, 3 \mathrm{H}) ;{ }^{13} \mathrm{C} \mathrm{NMR}\left(100 \mathrm{~Hz}, \mathrm{CDCl}_{3}\right): \delta$ 170.7, 170.2, 137.6, 129.3, 128.4, 128.0, 127.8, 127.7, 93.6, 70.2, 67.0, 65.2, 62.9, 20.9, 20.7. Anal. Calcd. for $\mathrm{C}_{17} \mathrm{H}_{20} \mathrm{O}_{6}(320.33)$ : C, 63.74; H, 6.29. Found: C, 63.50; H, 6.21\%.

2-Phenylethyl 4,6-di- $O$-acetyl-2,3-dideoxy- $\alpha$-D-erythro-hex-2-enopyranoside $\quad(3 c) . \quad[\alpha]_{\mathrm{D}}{ }^{25}$ $+76^{\circ}$ (c 0.16, $\mathrm{CH}_{2} \mathrm{Cl}_{2}$ ); IR (KBr): 3028, 2928, 1743, 1496, 1370, 1228, 1105, 1039, 972, 909, 751, 700, $669 \mathrm{~cm}^{-1} ;{ }^{1} \mathrm{H}$ NMR $\left(400 \mathrm{MHz}, \mathrm{CDCl}_{3}\right): \delta 7.23-7.18(\mathrm{~m}, 2 \mathrm{H}), 7.16-7.12(\mathrm{~m}, 3 \mathrm{H}), 5.81$ (dd, J.11.2 Hz, J.11.6 Hz, 1H), 5.75-5.71 (m, 1H), 5.21 (dd, J.1.6 Hz, J.9.6 Hz, 1H), 4.94 (s, 1H), 4.10 (dd, J.5.2 Hz, J.12 Hz, 1H), 3.96 (dd, J.2.8 Hz, J.12 Hz, 1H), 3.92-3.86 (m, 2H), 3.71-3.66 $(\mathrm{m}, 1 \mathrm{H}), 2.86(\mathrm{t}, 2 \mathrm{H}), 2.0(\mathrm{~s}, 3 \mathrm{H}), 1.99(\mathrm{~s}, 3 \mathrm{H}) ;{ }^{13} \mathrm{C} \mathrm{NMR}\left(100 \mathrm{~Hz}, \mathrm{CDCl}_{3}\right): \delta$ 170.9, 170.4, 
138.9, 129.2, 129.0, 128.5, 127.8, 126.4, 94.5, 69.6, 67.0, 65.3, 63.0, 36.4, 21.1, 20.9. Anal. Calcd. for $\mathrm{C}_{18} \mathrm{H}_{22} \mathrm{O}_{6}(334.36)$ : C, 64.66; H, 6.63. Found: C, 64.50; H, 6.51\%.

3-Phenylpropanyl 4,6-di- $O$-acetyl-2,3-dideoxy- $\alpha$-D-erythro-hex-2-enopyranoside $(3 \mathrm{~d})$. $[\alpha]_{\mathrm{D}}{ }^{25}$ $+81^{\circ}$ ( $c$ 0.28, $\mathrm{CH}_{2} \mathrm{Cl}_{2}$ ); IR (KBr): 3060, 3026, 2942, 1744, 1603, 1496, 1453, 1370, 1235, 1106, 1038, 978, 909, 746, 700, $605 \mathrm{~cm}^{-1} ;{ }^{1} \mathrm{H}$ NMR (400 MHz, $\left.\mathrm{CDCl}_{3}\right): \delta$ 7.22-7.07 (m, 5H), $5.81(\mathrm{~d}$, J.10.4 Hz, 1H), 5.78-5.74 (m, 1H), 5.23 (dd, J.9.2 Hz, J.1.6 Hz, 1H), 4.93 (s, 1H), 4.18-4.14 (m, $1 \mathrm{H}), 4.08-4.01(\mathrm{~m}, 2 \mathrm{H}), 3.75-3.69(\mathrm{~m}, 1 \mathrm{H}), 3.47-3.41(\mathrm{~m}, 1 \mathrm{H}), 2.67-2.57(\mathrm{~m}, 2 \mathrm{H}), 2.0(\mathrm{~s}, 3 \mathrm{H})$, $1.96(\mathrm{~s}, 3 \mathrm{H}), 1.89-1.85(\mathrm{~m}, 2 \mathrm{H}) ;{ }^{13} \mathrm{C} \mathrm{NMR}\left(100 \mathrm{~Hz}, \mathrm{CDCl}_{3}\right): \delta 170.8,170.3,141.7,129.1,128.5$, 128.0, 125.9, 94.6, 68.2, 67.0, 65.3, 63.0, 32.5, 31.3, 21.0, 20.8. Anal. Calcd. for $\mathrm{C}_{19} \mathrm{H}_{24} \mathrm{O}_{6}$ (348.39): C, 65.50; H, 6.94. Found: C, 65.40; H, 6.82\%.

4-Phenyl butanyl 4,6-di- $O$-acetyl-2,3-dideoxy- $\alpha$-D-erythro-hex-2-enopyranoside $(3 \mathrm{e}) \cdot[\alpha]_{\mathrm{D}}{ }^{25}$ $+54^{\circ}$ ( $c$ 0.22, $\mathrm{CH}_{2} \mathrm{Cl}_{2}$ ); IR (KBr): 3026, 2936, 2861, 1744, 1496, 1453, 1370, 1233, 1041, 748, 699, $669 \mathrm{~cm}^{-1} ;{ }^{1} \mathrm{H}$ NMR $\left(400 \mathrm{MHz}, \mathrm{CDCl}_{3}\right): \delta$ 7.29-7.13 (m, 5H), $5.87(\mathrm{~d}, J .11 .6 \mathrm{~Hz}, 1 \mathrm{H}), 5.83-$ $5.81(\mathrm{~m}, 1 \mathrm{H}), 5.30(\mathrm{dd}, J .9 .6 \mathrm{~Hz}, J .1 .2 \mathrm{~Hz}, 1 \mathrm{H}), 5.0(\mathrm{~s}, 1 \mathrm{H}), 4.25-4.19(\mathrm{~m}, 1 \mathrm{H}), 4.15$ (dd, J.12 Hz, J.2 Hz, 1H), 4.10-4.06 (m, 1H), 3.82-3.76 (m, 1H), 3.54-3.47 (m, 1H), 2.67-2.59 (m, 2H), 2.07 $(\mathrm{s}, 3 \mathrm{H}), 2.06(\mathrm{~s}, 3 \mathrm{H}), 1.72-1.62(\mathrm{~m}, 4 \mathrm{H}) ;{ }^{13} \mathrm{C} \mathrm{NMR}\left(100 \mathrm{~Hz}, \mathrm{CDCl}_{3}\right): \delta 170.8,170.3,142.3$, 129.0, 128.5, 128.4, 128.0, 125.8, 94.5, 68.8, 66.9, 65.3, 63.1, 35.7, 29.4, 28.2, 21.0, 20.8. Anal. Calcd. for $\mathrm{C}_{20} \mathrm{H}_{26} \mathrm{O}_{6}(362.41)$ : C, 66.28; H, 7.23. Found: C, 66.15; H, 7.14\%.

5-Phenyl pentyl 4,6-di- $\boldsymbol{O}$-acetyl-2,3-dideoxy- $\alpha$-D-erythro-hex-2-enopyranoside $(3 \mathrm{3}) \cdot[\alpha]_{\mathrm{D}}{ }^{25}$ $+12.8^{\circ}$ (c 0.40, $\mathrm{CH}_{2} \mathrm{Cl}_{2}$ ); IR (KBr): 3061, 3025, 2934, 2858, 1748, 1495, 1453, 1367, 1228, 1120, 1046, 907, 748, 700, $601 \mathrm{~cm}^{-1} ;{ }^{1} \mathrm{H}$ NMR (400 MHz, $\left.\mathrm{CDCl}_{3}\right): \delta 7.28-7.23(\mathrm{~m}, 2 \mathrm{H}), 7.17-$ $7.15(\mathrm{~m}, 3 \mathrm{H}), 5.94-5.83(\mathrm{~m}, 2 \mathrm{H}), 5.30(\mathrm{~d}, J .9 .2 \mathrm{~Hz}, 1 \mathrm{H}), 5.0(\mathrm{~s}, 1 \mathrm{H}), 4.22-4.17(\mathrm{~m}, 1 \mathrm{H}), 4.03-$ $3.99(\mathrm{~m}, 1 \mathrm{H}), 3.87-3.73(\mathrm{~m}, 1 \mathrm{H}), 3.52-3.44(\mathrm{~m}, 2 \mathrm{H}), 2.63-2.59(\mathrm{~m}, 2 \mathrm{H}), 2.07(\mathrm{~s}, 3 \mathrm{H}), 2.06(\mathrm{~s}$, $3 \mathrm{H}), 1.66-1.62(\mathrm{~m}, 4 \mathrm{H}), 1.44-1.39(\mathrm{~m}, 2 \mathrm{H}) .{ }^{13} \mathrm{C} \mathrm{NMR}\left(100 \mathrm{~Hz}, \mathrm{CDCl}_{3}\right): 171.0,170.5,142.7$, 129.2, 128.5, 128.1, 126.3, 125.9, 94.6, 69.0, 67.0, 65.5, 63.2, 36.0, 31.4, 29.8, 26.1, 21.2, 21.0. Anal. Calcd. for $\mathrm{C}_{21} \mathrm{H}_{28} \mathrm{O}_{6}(376.44)$ : C, 67.00; H, 7.50. Found: C, 66.85; H, 7.40\%.

n-Butyl 4,6-di- $O$-acetyl-2,3-dideoxy- $\alpha$-D-erythro-hex-2-enopyranoside $(\mathbf{3 g}) \cdot[\alpha]_{\mathrm{D}}{ }^{25}+106^{\circ}(c$ 0.23, $\mathrm{CH}_{2} \mathrm{Cl}_{2}$ ); IR (KBr): 2959, 2934, 2873, 1746, 1371, 1233, 1105, 1043, 907, 732, $605 \mathrm{~cm}^{-1}$; ${ }^{1} \mathrm{H}$ NMR (400 MHz, $\mathrm{CDCl}_{3}$ ): $\delta$ 5.82-5.75 ( m, 1H), 5.72-5.67 (m, 1H), $5.16(\mathrm{dd}, J .1 .2 \mathrm{~Hz}, J .9 .6$ $\mathrm{Hz}, 1 \mathrm{H}), 4.88$ (s, 1H), 4.10 (dd, J.5.6 Hz, J.12.4 Hz, 1H), 4.0 (dd, J.2.4 Hz, J.12 Hz, 1H), 3.98$3.94(\mathrm{~m}, 1 \mathrm{H}), 3.68-3.61(\mathrm{~m}, 1 \mathrm{H}), 3.40-3.33(\mathrm{~m}, 1 \mathrm{H}), 1.95(\mathrm{~s}, 3 \mathrm{H}), 1.94(\mathrm{~s}, 3 \mathrm{H}), 1.48-1.41$ (m, $2 \mathrm{H}), 1.29-1.22(\mathrm{~m}, 2 \mathrm{H}), 0.79(\mathrm{t}, 3 \mathrm{H}) ;{ }^{13} \mathrm{C} \mathrm{NMR}\left(100 \mathrm{~Hz}, \mathrm{CDCl}_{3}\right): \delta 170.6,170.2,128.9,127.9$, 94.3, 68.5, 66.8, 65.2, 63.0, 31.7, 20.8, 20.7, 19.3, 13.7. Anal. Calcd. for $\mathrm{C}_{14} \mathrm{H}_{22} \mathrm{O}_{6}$ (286.32): C, 58.73; H, 7.74. Found: C, 58.50; H, 7.65\%.

Octyl 4,6-di- $O$-acetyl-2,3-dideoxy- $\alpha$-D-erythro-hex-2-enopyranoside $(3 \mathrm{~h}) \cdot[\alpha]_{\mathrm{D}}{ }^{25}+16.6^{\circ}(c$ 0.62, $\mathrm{CH}_{2} \mathrm{Cl}_{2}$ ); IR (KBr): 3060, 3025, 2933, 2858, 1748, 1602, 1494, 1453, 1370, 1230, 1120, 1047, 906, 746, $700 \mathrm{~cm}^{-1}$; ${ }^{1} \mathrm{H}$ NMR (400 MHz, $\left.\mathrm{CDCl}_{3}\right): \delta 5.95-5.89(\mathrm{~m}, 1 \mathrm{H}), 5.87-5.85(\mathrm{~m}, 1 \mathrm{H})$, $5.31(\mathrm{~d}, J .9 .6 \mathrm{~Hz}, 1 \mathrm{H}), 5.03(\mathrm{~s}, 1 \mathrm{H}), 4.28-4.22(\mathrm{~m}, 1 \mathrm{H}), 4.19-4.16(\mathrm{~m}, 1 \mathrm{H}), 4.13-4.09(\mathrm{~m}, 1 \mathrm{H})$, 3.80-3.74 (m, 1H), 3.53-3.46 (m, 1H), $2.10(\mathrm{~s}, 3 \mathrm{H}), 2.08(\mathrm{~s}, 3 \mathrm{H}), 1.63-1.57(\mathrm{~m}, 5 \mathrm{H}), 1.32-1.29$ $(\mathrm{m}, 7 \mathrm{H}), 0.87(\mathrm{t}, J .5 .6 \mathrm{~Hz}, 3 \mathrm{H}) ;{ }^{13} \mathrm{C} \mathrm{NMR}\left(100 \mathrm{~Hz}, \mathrm{CDCl}_{3}\right): \delta 170.5,170.4,129.0,128.1,94.5$, 
69.1, 67.0, 65.4, 63.1, 31.9, 29.8, 29.5, 29.4, 26.3, 22.7, 21.0, 20.9, 14.2. Anal. Calcd. for $\mathrm{C}_{18} \mathrm{H}_{30} \mathrm{O}_{6}$ (342.42): C, 63.14; H, 8.83. Found: C, 62.95; H, 8.70\%.

Hexadecyl 4,6-di- $O$-acetyl-2,3-dideoxy- $\alpha$-D-erythro-hex-2-enopyranoside $(3 i) \cdot[\alpha]_{\mathrm{D}}^{25}+50^{\circ}(c$ $0.07, \mathrm{CH}_{2} \mathrm{Cl}_{2}$ ); IR (KBr): 2924, 2853, 1748, 1466, 1369, 1233, 1105, $1040 \mathrm{~cm}^{-1} ;{ }^{1} \mathrm{H}$ NMR (400 $\left.\mathrm{MHz}, \mathrm{CDCl}_{3}\right): \delta 5.88(\mathrm{~d}, J .10 .4 \mathrm{~Hz}, 1 \mathrm{H}), 5.85-5.82(\mathrm{~m}, 1 \mathrm{H}), 5.31(\mathrm{dd}, J .10 \mathrm{~Hz}, J .1 .2 \mathrm{~Hz}, 1 \mathrm{H})$, $5.02(\mathrm{~s}, 1 \mathrm{H}), 4.25$ (dd, J.12 Hz, J.5.2 Hz, 1H), 4.17 (dd, J.12 Hz, J.2.4 Hz, 1H), 4.12-4.09 (m, $1 \mathrm{H}), 3.78-3.73(\mathrm{~m}, 1 \mathrm{H}), 3.53-3.47(\mathrm{~m}, 1 \mathrm{H}), 2.10(\mathrm{~s}, 3 \mathrm{H}), 2.08(\mathrm{~s}, 3 \mathrm{H}), 1.63-1.59(\mathrm{~m}, 4 \mathrm{H}), 1.25-$ $1.20(\mathrm{~m}, 24 \mathrm{H}), 0.88(\mathrm{t}, J .6 .34,3 \mathrm{H}) ;{ }^{13} \mathrm{C} \mathrm{NMR}\left(100 \mathrm{~Hz}, \mathrm{CDCl}_{3}\right): \delta 170.8,170.3,129.2,128.1$, 94.6, 69.2, 67.0, 65.5, 63.3, 32.1, 29.8 (9C), 29.6 (2C), 26.4, 22.9, 21.2, 21.0, 14.3. Anal. Calcd. for $\mathrm{C}_{26} \mathrm{H}_{46} \mathrm{O}_{6}$ (454.64): C, 68.69; H, 10.20. Found: C, 68.55; H, $10.05 \%$.

Heptyl 4,6-di- $O$-acetyl-2,3-dideoxy- $\alpha$-D-erythro-hex-2-enopyranoside $(3 \mathbf{j}) \cdot[\alpha]_{\mathrm{D}}{ }^{25}+68^{\mathrm{o}}(c$ $0.15, \mathrm{CH}_{2} \mathrm{Cl}_{2}$ ); IR (KBr): 2928, 2857, 1745, 1369, 1234, 1105, 1039, 908, $802 \mathrm{~cm}^{-1} ;{ }^{1} \mathrm{H} \mathrm{NMR}$ (400 MHz, $\left.\mathrm{CDCl}_{3}\right): \delta 5.98-5.83(\mathrm{~m}, 2 \mathrm{H}), 5.33(\mathrm{dd}, J .1 .2 \mathrm{~Hz}, J .11 .2 \mathrm{~Hz}, 1 \mathrm{H}), 5.04(\mathrm{~s}, 1 \mathrm{H}), 4.35-$ 4.29 (m, 1H), 4.25 (dd, J.2.4 Hz, J.12 Hz, 1H), 3.87-3.81 (m, 1H), 3.61-3.48 (m, 2H), 2.18 (s, $3 \mathrm{H}), 2.16(\mathrm{~s}, 3 \mathrm{H}), 1.70-1.63(\mathrm{~m}, 3 \mathrm{H}), 1.38-1.36(\mathrm{~m}, 7 \mathrm{H}), 0.95(\mathrm{t}, 3 \mathrm{H}) .{ }^{13} \mathrm{C} \mathrm{NMR}\left(100 \mathrm{~Hz}, \mathrm{CDCl}_{3}\right)$ : $\delta 170.9,170.4,129.1,128.2,94.5,69.1,67.0,65.5,63.2,31.9,29.8,29.2,26.3,22.7,21.1,20.9$, 14.2. Anal. Calcd. for $\mathrm{C}_{17} \mathrm{H}_{28} \mathrm{O}_{6}$ (328.40): C, 62.17; H, 8.59. Found: C, 62.02; H, 8.44\%.

Isopropyl 4,6-di- $O$-acetyl-2,3-dideoxy- $\alpha$-D-erythro-hex-2-enopyranoside $(3 \mathbf{k})$. $[\alpha]_{\mathrm{D}}{ }^{25}+112^{\circ}(c$ 0.18, $\mathrm{CH}_{2} \mathrm{Cl}_{2}$ ); IR (KBr): 2972, 2931, 1745, 1453, 1371, 1236, 1184, 1125, 1100, 1036, 982, 909, 731, $606 \mathrm{~cm}^{-1} ;{ }^{1} \mathrm{H}$ NMR (400 MHz, $\left.\mathrm{CDCl}_{3}\right): \delta 5.82-5.73(\mathrm{~m}, 2 \mathrm{H}), 5.23(\mathrm{~d}, J .10 \mathrm{~Hz}, 1 \mathrm{H})$, 5.07 (s, 1H), 4.18 (dd, J.6 Hz, J.12 Hz, 2H), 4.14-4.07 (m, 1H), 3.96-3.90 (m, 1H), 2.03 (s, 3H), $2.02(\mathrm{~s}, 3 \mathrm{H}), 1.20(\mathrm{~d}, J .6 \mathrm{~Hz}, 3 \mathrm{H}), 1.12(\mathrm{~d}, J .6 \mathrm{~Hz}, 3 \mathrm{H}) ;{ }^{13} \mathrm{C} \mathrm{NMR}\left(100 \mathrm{~Hz}, \mathrm{CDCl}_{3}\right): \delta 170.8$, $170.3,128.8,128.5,92.9,70.8,66.8,65.5,63.2,23.6,22.0,21.0,20.8$. Anal. Calcd. for $\mathrm{C}_{13} \mathrm{H}_{20} \mathrm{O}_{6}$ (272.29): C, 57.34; H, 7.40. Found: C, 57.20; H, 7.25\%.

tert-Butyl 4,6-di- $\boldsymbol{O}$-acetyl-2,3-dideoxy- $\alpha$-D-erythro-hex-2-enopyranoside $(3 \mathrm{l}) \cdot[\alpha]_{\mathrm{D}}{ }^{25}+102^{\circ}(c$ 0.14, $\mathrm{CH}_{2} \mathrm{Cl}_{2}$ ); IR (KBr): 2976, 2933, 1746, 1437, 1369, 1235, 1195, 1099, 1044, 982, 891, 774, 716, $605 \mathrm{~cm}^{-1} ;{ }^{1} \mathrm{H}$ NMR (400 MHz, $\left.\mathrm{CDCl}_{3}\right): \delta 5.84(\mathrm{~d}, J .10 \mathrm{~Hz}, 1 \mathrm{H}), 5.77-5.73(\mathrm{~m}, 1 \mathrm{H}), 5.32(\mathrm{~s}$, 1H), $5.27(\mathrm{~d}, J .8 .8 \mathrm{~Hz}, 1 \mathrm{H}), 4.26-4.21(\mathrm{~m}, 1 \mathrm{H}), 4.19-4.13(\mathrm{~m}, 2 \mathrm{H}), 2.08(\mathrm{~s}, 3 \mathrm{H}), 2.07$ (s, 3H), 1.29 (s, 9H); ${ }^{13} \mathrm{C}$ NMR $\left(100 \mathrm{~Hz}, \mathrm{CDCl}_{3}\right): \delta 171.0,170.6,129.7,128.3,89.1,75.5,66.7,65.5$, 63.4, 28.9, 21.1, 21.0. Anal. Calcd. for $\mathrm{C}_{14} \mathrm{H}_{22} \mathrm{O}_{6}$ (286.32): C, 58.73; H, 7.74. Found: C, 58.55; H, $7.57 \%$.

Allyl 4,6-di- $\boldsymbol{O}$-acetyl-2,3-dideoxy- $\alpha$-D-erythro-hex-2-enopyranoside $(\mathbf{3 m}) \cdot[\alpha]_{\mathrm{D}}{ }^{25}+102^{\circ}(c$ 0.12, $\mathrm{CH}_{2} \mathrm{Cl}_{2}$ ); IR (KBr): 3081, 2924, 1742, 1652, 1370, 1229, 1101, 1039, 812, 743, $603 \mathrm{~cm}^{-1}$; ${ }^{1} \mathrm{H}$ NMR (400 MHz, $\left.\mathrm{CDCl}_{3}\right): \delta 5.94-5.81(\mathrm{~m}, 3 \mathrm{H}), 5.30-5.24(\mathrm{~m}, 2 \mathrm{H}), 5.18-5.15(\mathrm{~m}, 1 \mathrm{H}), 5.04(\mathrm{~s}$, 1H), 4.24-4.19 (m, 2H), 4.14 (dd, J.2.4 Hz, J.12.4 Hz, 1H), 4.10-4.02 (m, 2H), 2.06 (s, 3H), 2.04 (s, 3H); ${ }^{13} \mathrm{C}$ NMR $\left(100 \mathrm{~Hz}, \mathrm{CDCl}_{3}\right): \delta 170.9,170.4,134.2,129.3,127.9,117.6,93.7,69.4,67.0$, 65.4, 63.0, 21.0, 20.9. Anal. Calcd. for $\mathrm{C}_{13} \mathrm{H}_{18} \mathrm{O}_{6}$ (270.28): C, 57.77; H, 6.71. Found: C, 57.59; H, $6.78 \%$.

Propynyl 4,6-di- $O$-acetyl-2,3-dideoxy- $\alpha$-D-erythro-hex-2-enopyranside $(3 n) \cdot[\alpha]_{\mathrm{D}}{ }^{25}+161^{\circ}(c$ 0.18, $\mathrm{CH}_{2} \mathrm{Cl}_{2}$ ); IR (KBr): 3465, 3278, 2917, 1742, 1373, 1229, 1141, 1099, 1039, 965, 909, 731, 
680, $613 \mathrm{~cm}^{-1} ;{ }^{1} \mathrm{H}$ NMR (400 MHz, $\left.\mathrm{CDCl}_{3}\right): \delta 5.92(\mathrm{~d}, J .10 .4 \mathrm{~Hz}, 1 \mathrm{H}), 5.86-5.83(\mathrm{~m}, 1 \mathrm{H}), 5.33$ (dd, J.1.2 Hz, J.9.6 Hz, 1H), 5.24 (s, 1H), 4.31 (d, J.2.4 Hz, 2H), 4.23 (d, J.4.8 Hz, 1H), 4.18 (dd, J.2.4 Hz, J.12.4 Hz, 1H), 4.10-4.07 (m, 1H), 2.52 (t, 1H), $2.10(\mathrm{~s}, 3 \mathrm{H}), 2.09$ (s, 3H); ${ }^{13} \mathrm{C} \mathrm{NMR}$ $\left(100 \mathrm{~Hz}, \mathrm{CDCl}_{3}\right): \delta 170.6,170.1,129.7,127.2,92.6,79.0,74.9,67.1,65.0,62.7,54.9,20.8,20.7$. Anal. Calcd. for $\mathrm{C}_{13} \mathrm{H}_{16} \mathrm{O}_{6}(268.26)$ : C, 58.20; H, 6.01. Found: C, 58.01; H, 5.94\%.

\section{Acknowledgements}

RSB and ML are thankful to CSIR, New Delhi, India for their research fellowships. The authors are grateful to the Director, IIT Guwahati for providing the general facilities to carry out this work. We acknowledge DST, New Delhi, for providing single crystal XRD facility to the Department of Chemistry under FIST programme (S. No.: SR/FST/CSII-007/2003). We are thankful to the referees for their valuable comments and suggestions.

\section{Supporting Information}

Supplementary data (spectral data of all compounds and copies of ${ }^{1} \mathrm{H},{ }^{13} \mathrm{C}$ NMR and of products) associated with this article can be found with the online version.

\section{References}

1. (a) Friesen, R. W.; Danishefsky, S. J. J. Am. Chem. Soc. 1989, 111, 6656. (b) Bilodeau, M. T.; Danishefsky, S. J. In Modern Methods in Carbohydrate Synthesis; Khan, S. H.; O’Neill, R. A. Eds.; Hardwood Academic Publishers GmbH: Amsterdam, 1996, pp 171-193. (c) Bussolo, V. D.; Kim, Y. J.; Gin, D. Y. J. Am. Chem. Soc. 1998, 120, 13515.

2. (a) Postema M. H. D. C-Glycosides Synthesis; CRC Press: London, 1995, pp $43-57$ (b) Cusk, R.; Schaade, M.; Krieger, C. Tetrahedron 1996, 52, 6397. (c) Takhi, M.; Rahman, A.; Schmidt, R. R. Tetrahedron Lett. 2001, 42, 4053.

3. (a) Tanimoto, H.; Saito, R.; Chida, N. Tetrahedron Lett. 2008, 49, 358. (b) Sabitha, G.; Reddy, S. S. S.; Yadav, J. S. Tetrahedron Lett. 2010, 51, 6259.

4. (a) Ferrier, R. J.; Ciment, D. M. J. Chem. Soc. C 1966, 441. (b) Ferrier, R. J.; Prasad, N. J. Chem. Soc. C 1969, 570. (c) Ferrier, R. J.; Prasad, N. J. Chem. Soc. C 1969, 581. (d) Ferrier, R. J. Adv. Carbohydr. Chem. Biochem. 1969, pp 199-266. (e) Ferrier R. J.; Zubkov, O. A. Org. React. 2003, 62, 569 and references therein (f) Ferrier R. J. Top. Curr. Chem. 2001, 215, 153. (g) Descotes, G.; Martin, J. C. Carbohydr. Res, 1977, 56, 168. (h) Klaffke, W.; Pudlo, P.; Springer, D.; Thieme, J. Liebigs Ann. Chem. 1991, 509. 
5. Williams, N. R.; Wander, J. D. The Carbohydrates, Chemistry and Biochemistry; Academic Press: New York, 1980; pp. 761.

6. López, J. C.; Gómez, A. M.; Valverde, S.; Fraser-Reid, B. J. Org. Chem. 1995, 60, 3851.

7. Gorityala, B. K.; Cai, S.; Lorpitthaya, R.; Ma, J.; Pasunooti, K. K.; Liu, X-W. Tetrahedron Lett. 2009, 50, 676.

8. (a) Yadav, J. S.; Reddy, B. V. S.; Pandey, S. K. New J. Chem. 2001, 25, 538. (b) Babu, B. S.; Balasubramanian, K. K. Tetrahedron Lett. 2000, 41, 1271. (c) Masson, C.; Soto, J.; Bessodes, M. Synlett 2000, 1281. (d) Yadav, J. S.; Reddy, V. S.; Reddy, K. B.; Satyanarayana, M. Tetrahedron Lett. 2002, 43, 7009. (e) Zhang, G.; Liu, Q.; Shi, L.; Wang, J. Tetrahedron 2008, 64, 339. (f) Hotha, S.; Tripathi, A. Tetrahedron Lett. 2005, 46, 4555.

9. (a) Yadav, J. S.; Reddy, B. V.; Murthy, C. V.; Kumar, G. M. Synlett 2000, 1450. (b) AbdelRahman, A. A.-H.; Winterfeld, G. A.; Takhi, M.; Schmidt, R. R. Eur. J. Org. Chem. 2002, 713. (c) Yadav, J. S.; Subba Reddy, B. V.; Sunder Reddy, J. S. J. Chem. Soc., Perkin Trans. 1 2002, 2390. (d) Babu, J. L.; Khare, A.; Vankar, Y. D. Molecules 2005, 10, 884.

10. (a) Agarwal, A.; Rani, S.; Vankar, Y. D. J. Org. Chem. 2004, 69, 6137. (b) Kinfe, H. H.; Mebrahtu, F. M.; Sithole, K. Carbohydr. Res. 2011, 346, 2528. (c) Levecque, P.; Gammon, D. W.; Jacobs, P.; De Vos, D.; Sels, B. Green Chem. 2010, 12, 828. (d) Gorityala, B. K.; Lorpitthaya, R.; Bai, Y.; Liu, X.-W. Tetrahedron 2009, 65, 5844. (e) de Oliveira, R. N.; Filho, J. R. d. F.; Srivastava, R. M. Tetrahedron Lett. 2002, 43, 2141; (f) Toshima, K.; Miyamoto, N.; Matsuo, G.; Nakata, M.; Matsumura, S. Chem Commun. 1996, 1379. (g) Rafiee, E.; Tangestaninejad, S.; Habibi, M. H.; Mirkhani, V. Bioorg. Med. Chem. Lett. 2004, 14, 3611. (h) Shanmugasundaram, B.; Bose, A. K.; Balasubramanian, K. K. Tetrahedron Lett. 2002, 43, 6795.

11. Xiong, D.-C.; Zhang, L.-H.; Ye, X.-S. Adv. Synth. Catal. 2008, 350, 1696.

12. (a) Khan, A. T.; Khan, M. M. Carbohydrate Research, 2010, 345, 154; (b) Khan, A. T.; M. M. Khan, Carbohydrate Research, 2010, 345, 2139.

13. Choudhury, L.H.; Parvin, T.; Khan, A.T. Tetrahedron 2009, 65, 9513.

14. (a) Khan, A. T.; Basha, R. S.; Lal, M. Tetrahedron Lett. 2012, 53, 2211 (b) Khan, A. T.; Ali, S.; Dar, A. A.; Lal, M. Tetrahedron Lett. 2011, 52, 5157. (c) Khan, A. T.; Basha, R. S.; Lal, M.; Mir, M. H. RSC. Adv. 2012, 2, 5506. 